\title{
Development and validation of a prognostic signature for preoperative prediction of overall survival in gastric cancer patients
}

This article was published in the following Dove Press journal: OncoTargets and Therapy

Minggu Zhu',*

Qicai Wang ${ }^{2, *}$

Zhaowen Luo'

Kelong Liu ${ }^{2}$

Zhiqiao Zhang'

'Department of Internal Medicine, The Affiliated Chencun Hospital of Shunde Hospital, Southern Medical University, Shunde District, Guangdong, China; ${ }^{2}$ Department of General Surgery, The Affiliated Chencun Hospital of Shunde Hospital, Southern Medical University, Shunde District, Guangdong, China

*These authors contributed equally to this work
Correspondence: Zhiqiao Zhang Department of Internal Medicine, The Affiliated Chencun Hospital of Shunde Hospital, Southern Medical University, No. 2 Anning Road, Chencun Town, 5283 I3, Shunde District, Foshan City, Guangdong Province, China Email sdgrxjbk@163.com
Background: As a serious challenge for public health, the prognosis of gastric cancer patients is still poor. The current study aimed to develop and validate a prognostic signature to predict the overall survival of gastric cancer patients.

Patients and methods: The dataset in the present study was obtained from The Cancer Genome Atlas database. The present study finally included 343 gastric cancer patients with information on long non-coding RNA (lncRNA) expression and overall survival.

Results: A prognostic model named Eleven-lncRNA signature was constructed according to the expression values of eleven prognostic lncRNA predictors identified by univariate and multivariate Cox regression model. According to time-dependent receiver operating characteristic curves, the Harrell's concordance indexes of Eleven-lncRNA signature were 0.764 (95\% CI $0.720-0.808), 0.776$ (95\% CI 0.732-0.820), and 0.807 (95\% CI 0.763-0.851) for 1-year overall survival, 3-year overall survival, and 5-year overall survival respectively in the model group. In the validation group, the Harrell's concordance indexes of Eleven-lncRNA signature were 0.748 (95\% CI 0.704-0.792), 0.794 (95\% CI 0.750-0.838), and 0.798 (95\% CI 0.754-0.842) for 1-year overall survival, 3-year overall survival, and 5-year overall survival respectively. The gastric cancer patients $(n=343)$ in the model group could be stratified into low-risk group $(\mathrm{n}=171)$ and high-risk group $(\mathrm{n}=172)$ according to the median of Eleven-lncRNA signature score. Kaplan-Meier survival curves showed that the mortality rate in the high-risk group was significantly poorer than that in the low-risk group $(P<0.001)$.

Conclusion: The present study constructed and validated a prognostic model named ElevenlncRNA signature for preoperative individual mortality risk prediction in gastric cancer patients. This Eleven-lncRNA signature can predict the individual mortality risk of gastric cancer patients and is helpful in improving clinical decision making regarding individualized treatment.

Keywords: long non-coding RNA, gastric cancer, overall survival, signature, prognosis

\section{Background}

Gastric cancer (GC) is still a serious challenge for public health, as the fourth most common malignant tumor and the second leading cause of cancer-related death worldwide. ${ }^{1}$ Despite the advances in early diagnosis and clinical treatment, the prognosis of GC patients is still poor. ${ }^{2,3}$ In clinical practice, most GC patients are diagnosed at advanced stage accompanied with distant metastasis or tumor invasion, and subsequently have a poor overall survival. ${ }^{4,5}$ The TNM staging system has been used to predict the prognosis of GC patients, but is limited by inadequate prognostic information and predictive ability for clinical management. ${ }^{6,7}$ Therefore, a reliable prognostic 
signature is needed to discriminate the high-risk patients from the low-risk patients and optimize personalized treatment decision making.

Long non-coding RNAs (lncRNAs) are a class of RNAs with a length of $>200$ nucleotides and no protein-coding function. ${ }^{8}$ It has been reported that $\operatorname{lncRNAs}$ are related to prognosis of GC patients and might serve as prognostic biomarkers for GC patients. ${ }^{9,10}$ Recently, several prognostic signatures have been constructed for prediction of overall survival in GC patients based on lncRNA expression values. ${ }^{1-14}$ However, these prognostic signatures have limitations due to the following reasons. First, these signatures were too complicated to calculate, for clinicians and patients. Second, the original lncRNA expression values in these signatures were generated on special gene detection platforms and subsequently reduced the repeatability of prognostic signatures on other detection platforms. Third, the original lncRNA expression values in these signatures were further standardized by different standardization methods. For example, the IncRNA expression values were standardized by Robust Multichip Average algorithm in studies performed by Song et al and Fan et al. ${ }^{12,13}$ The special transformation methods used on original lncRNA expression values further reduced the clinical application of these prognostic signatures, because these special standardization methods used in previous studies might not be available to clinicians and patients. Therefore, there is a strong need for prognostic signatures which are more efficient, more accurate, and easier to calculate for clinicians and patients.

Nomogram has been recommended for predicting the prognosis in various cancers. ${ }^{10,15}$ Nomogram is easier to calculate and transform to a normalized individual mortality probability, thus, the individual mortality risk is easy to understand for patients without professional medical knowledge. The critical goal of the present study was to develop and validate a prognostic signature for overall survival of GC patients. We performed the current study according to the guidelines of Transparent Reporting of a multivariable prediction model for Individual Prognosis Or Diagnosis (TRIPOD). ${ }^{16}$

\section{Methods}

\section{Protocol approval}

The original dataset in the present study was downloaded from The Cancer Genome Atlas (TCGA) database. We downloaded and analyzed the study data in accordance with the relevant data policies of TCGA database and therefore no additional ethics approval was needed.

\section{The gene expression dataset}

The original study dataset was obtained from TCGA data portal (up to April 1, 2018, https://tcga-data.nci.nih.gov/ docs/publications/tcga/). The original RNA expression values were generated on the Illumina HiSeq 2000 RNA Sequencing platform. There were 14,449 lncRNAs from 375 GC tissues and 32 normal tissues in the original study dataset.

\section{Differential expression analyses}

The original lncRNA expression counts $<1$ were filtered out from the current study. The original lncRNA expression counts were normalized using Trimmed Mean of $\mathrm{M}$ method with "edgeR" package. ${ }^{17} \mid \log _{2}$ fold change $\mid>2$ and $P$-value $<0.05$ were defined as the thresholds for differential expression analyses.

\section{Clinical dataset}

The clinical dataset of $443 \mathrm{GC}$ patients was obtained from TCGA data portal (up to April 1, 2018, https://tcga-data.nci. nih.gov/docs/publications/tcga/). To avert the influences of confounding factors, 41 patients were excluded from the current study due to inadequate overall survival information (defined as overall survival time shorter than 1 month). There were 59 patients excluded from the clinical dataset due to inadequate lncRNA expression information. As shown in Figure 1, there were 343 GC patients included in the present study for final survival analysis. The maximum survival time was 122.2 months and the minimum survival time was 1.0 month. The study form completion date was from July 22, 2010, to April 16,2015 . We coded missing data as "NA" (not available) in the present study. The mean $\pm \mathrm{SD}$ age of GC patients in the model cohort was $65.5 \pm 10.6$ years. One hundred and forty-six $(42.6 \%)$ patients out of $343 \mathrm{GC}$ patients died within the follow-up period (mean \pm SD: $624 \pm 537$ days).

\section{Internal validation}

To perform an internal validation, we constructed a validation dataset using the bootstrap resampling method, which is recommended for validation of a prognostic model. ${ }^{18,19}$ The validation dataset in the current study was built by drawing 343 GC patients with replacements from the original model dataset.

\section{Statistical analysis}

Continuous variables were displayed as mean \pm SD. Continuous variables were compared by Student's $t$-test or Mann-Whitney $\mathrm{U}$ test as appropriate. Categorical variables were compared by using chi-squared test or Fisher's exact test as appropriate. The predictive performance and clinical utility of prognostic 


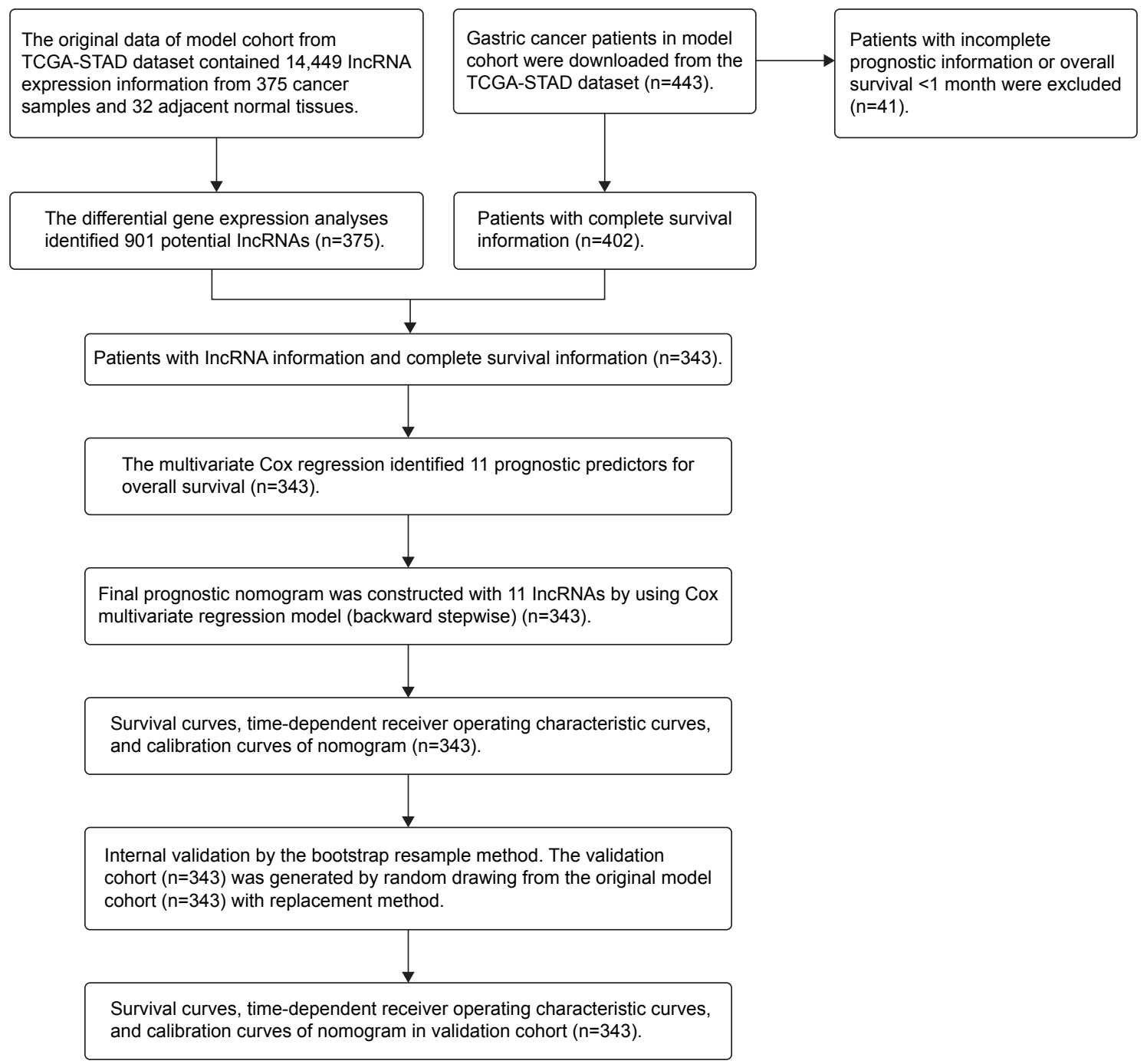

Figure I Flowchart of the current study.

Abbreviations: IncRNA, long non-coding RNA; TCGA, The Cancer Genome Atlas; STAD, stomach adenocarcinoma.

signatures were evaluated by using the Harrell's concordance index (C-index) and time-dependent receiver operating characteristic (ROC) curves. The statistical analyses in the present study were performed using SPSS 19.0 (IBM Corporation, Armonk, NY, USA) and R software (version 3.4.4). The R packages, including "pROC", "plyr", "rms", "survival", "timeROC", and "glmnet" were used as needed in the current study. The criterion for statistical significance was $P<0.05$.

\section{Results}

\section{Study cohort}

There were 343 GC patients with lncRNA expression information and prognosis information in the current study. The average age of $343 \mathrm{GC}$ patients was $65.5 \pm 10.6$ years and the average survival time was $20.8 \pm 17.9$ months in the present study. One hundred and forty-six (42.6\%) patients out of
343 patients died within the follow-up period in the present study. The basic clinical characteristics of GC patients in the model group and validation group were presented in Table 1. The differences in clinical characteristics were not significant between model group and validation group.

\section{Differential expression analyses}

The differential expression analyses were carried out between 375 cancer tissues and 32 normal tissues. Nine hundred and one differential lncRNAs were identified by "edgeR" package. The heat map (Figure S1) and volcano map (Figure S2) are provided in the Supplementary materials.

\section{Construction of prognostic signature}

The univariate and multivariate Cox proportional regression analyses were carried out to identify potential lncRNA 
Table I The clinical features of gastric cancer patients in model group and validation group

\begin{tabular}{l|l|l|l}
\hline & Model group (n=343) & Validation group (n=343) & P-value \\
\hline Death, $n$ (\%) & $146(42.6)$ & $151(44.0)$ & 0.70 \\
Survival time (mean \pm SD, months) & $20.8 \pm 17.9$ & $21.2 \pm 17.6$ & 0.450 \\
Age (mean \pm SD, years) & $65.5 \pm 10.6$ & $65.8 \pm 10.4$ & 0.561 \\
Gender (male/female) & $224 / 119$ & $219 / 124$ & 0.690 \\
AJCC stage (IV/III/II//NA) & $34 / 140 / 107 / 47 / / 5$ & $25 / 139 / 109 / 51 / 19$ & 0.359 \\
AJCC PT (T4/T3/T2/TI/NA) & $93 / 159 / 73 / 18 / 0$ & $80 / 167 / 67 / 29 / 0$ & 0.261 \\
AJCC PN (N3/N2/NI/NO/NA) & $78 / 69 / 94 / 100 / 2$ & $81 / 71 / 84 / 104 / 3$ & 0.915 \\
AJCC PM (MX/MI/MO/NA) & $14 / 22 / 307 / 0$ & $16 / 14 / 3 / 3 / 0$ & 0.474 \\
Radiation treatment adjuvant (yes/no/NA) & $0 / 186 / 157$ & $0 / 185 / 158$ & 0.939 \\
Pharmaceutical adjuvant (yes/no/NA) & $43 / 148 / 152 / 0$ & $42 / 147 / 154 / 0$ & 0.986 \\
History of other malignance (yes/no/NA) & $8 / 336 / 0$ & $9 / 335 / 0$ & 0.806 \\
\hline
\end{tabular}

Note: Continuous variables were compared by Student's $t$-test or Mann-Whitney $U$ test as appropriate; categorical variables were compared by chi-squared test or Fisher's exact test as appropriate.

Abbreviations: AJCC, American Joint Committee on Cancer; NA, not available.

predictors for overall survival. The multivariate Cox regression analysis finally identified eleven potential lncRNA predictors for overall survival. The relevant model information of eleven lncRNAs in Cox regression analysis was presented in Table 2. A prognostic signature (Figure 2) was constructed according to the expression values of eleven lncRNA predictors for overall survival of GC patients: Eleven-lncRNA signature score $=\left(0.5648^{*} \mathrm{RP} 11-61011.1\right)+\left(-0.8865^{*}\right.$ RP11-766N7.3)+(0.6886* AC027119.1)+(-0.6076* RP5$881 \mathrm{~L} 22.5)+\left(0.618^{*}\right.$ RP11-332J15.4 $)+\left(-0.5248^{*}\right.$ RP11-616M22.7)+(-0.7319* AC002463.3)+(0.6338* ERICH3-AS 1$)+(0.5576$ * ZFPM2-AS1 $)+(-0.5482$ * HOXA11-AS $)+\left(-0.5055^{*}\right.$ RP11-319G6.3).

\section{Prognostic value of Eleven-IncRNA signature}

All GC patients $(n=343)$ in the model group (Table S1) were stratified into high-risk group $(\mathrm{n}=172)$ and low-risk group ( $\mathrm{n}=171)$ according to the median of Eleven-lncRNA signature score. The overall survival rate (Figure 3A) in the high-risk group was significantly poorer than that in the lowrisk group $(P<0.001)$. The distribution of Eleven-lncRNA signature score (Figure 3B), survival status and survival time (Figure $3 \mathrm{C}$ ) in the model group were presented in Figure 3. The Harrell's C-index of Eleven-lncRNA signature for overall survival was 0.734 (95\% CI: $0.690-0.778)$ in the model group.

\section{Clinical utility of Eleven-IncRNA signature}

According to time-dependent ROC curves, the C-indexes of Eleven-lncRNA signature were 0.764, 0.776, and 0.807 for 1-year overall survival, 3-year overall survival, and 5-year overall survival respectively (Figure 4A). A good agreement between predictive mortality probability and actual mortality percentage was found through calibration curves of 1-year overall survival (Figure 4B), 3-year

Table 2 The model information of eleven prognostic IncRNA predictors in univariate and multivariable Cox regression analyses

\begin{tabular}{|c|c|c|c|c|c|c|c|}
\hline \multirow[t]{2}{*}{ Variables } & \multicolumn{3}{|c|}{ Univariate analysis } & \multicolumn{4}{|c|}{ Multivariate analysis } \\
\hline & HR & $95 \% \mathrm{Cl}$ & $P$-value & Coefficient & HR & $95 \% \mathrm{Cl}$ & $P$-value \\
\hline RPII-6IOII.I (high/low) & 1.628 & $|| 72-2.26 \mid$. & 0.004 & 0.5648 & 1.759 & $1.243-2.490$ & 0.5648 \\
\hline RPII-766N7.3 (high/low) & 0.629 & $0.453-0.873$ & 0.006 & -0.8865 & 0.412 & $0.288-0.589$ & -0.8865 \\
\hline AC027II9.I (high/low) & 1.625 & $1.169-2.258$ & 0.004 & 0.6886 & 1.991 & $1.399-2.833$ & 0.6886 \\
\hline RP5-88IL22.5 (high/low) & 0.663 & $0.476-0.923$ & 0.015 & -0.6076 & 0.545 & $0.38 \mathrm{I}-0.780$ & -0.6076 \\
\hline RPII-332JI5.4 (high/low) & 1.565 & $1.123-2.181$ & 0.008 & 0.618 & 1.855 & $1.305-2.637$ & 0.618 \\
\hline RPII-6I6M22.7 (high/low) & 0.692 & $0.496-0.965$ & 0.030 & -0.5248 & 0.592 & $0.421-0.833$ & -0.5248 \\
\hline AC002463.3 (high/low) & 0.690 & $0.497-0.959$ & 0.027 & -0.7319 & 0.481 & $0.336-0.688$ & -0.7319 \\
\hline ERICH3-ASI (high/low) & 1.541 & $1.108-2.145$ & 0.010 & 0.6338 & 1.885 & $1.319-2.692$ & 0.6338 \\
\hline ZFPM2-ASI (high/low) & 1.403 & $1.012-1.944$ & 0.042 & 0.5576 & 1.747 & $1.238-2.464$ & 0.5576 \\
\hline HOXAII-AS (high/low) & 0.702 & $0.506-0.973$ & 0.034 & -0.5482 & 0.578 & $0.407-0.822$ & -0.5482 \\
\hline RPII-3I9G6.3 (high/low) & 0.719 & $0.518-0.998$ & 0.048 & -0.5055 & 0.603 & $0.430-0.846$ & -0.5055 \\
\hline
\end{tabular}

Note: The medians of IncRNA expression values were used as cutoff values to stratify IncRNA expression values into high expression group (as value I) and low expression group (as value 0).

Abbreviation: IncRNA, long non-coding RNA. 


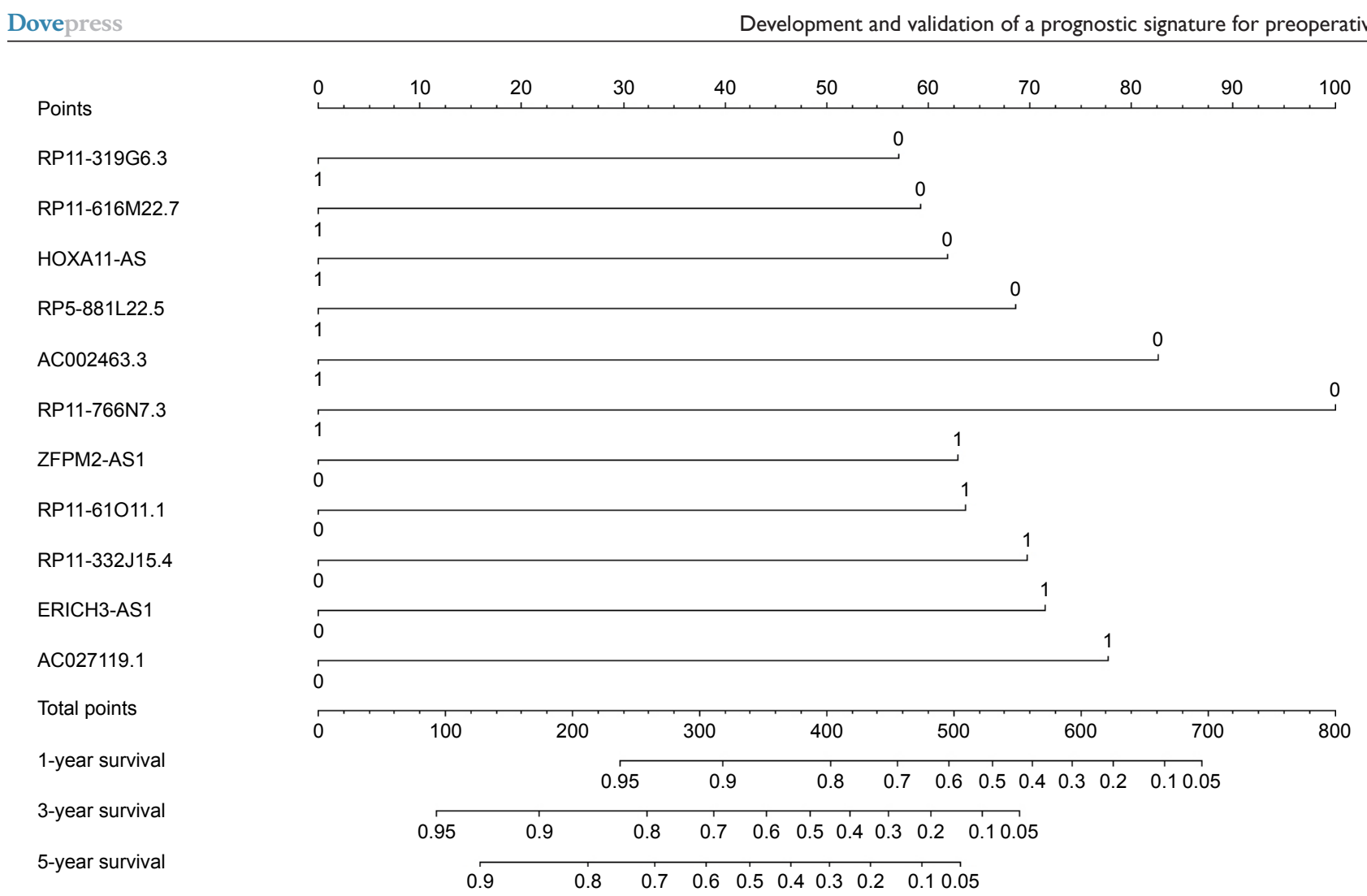

Figure 2 The Eleven-IncRNA signature for prediction of overall survival in gastric cancer patients. Abbreviation: IncRNA, long non-coding RNA.

overall survival (Figure 4C), and 5-year overall survival (Figure 4D).

\section{Validation of Eleven-IncRNA signature}

We built an internal validation dataset (Table S2 [n=343]) for internal validation by using random drawing with replacement method. The Eleven-lncRNA signature scores of patients in the validation group were calculated according to the original formula of Eleven-lncRNA signature in the model group. The GC patients $(n=343)$ in validation group were divided into high-risk group and low-risk group according to the previous cutoff value in the model group. The log-rank test (Figure 5A) demonstrated that the mortality rate in the high-risk group was significantly poorer than that in the low-risk group $(P<0.001)$. The distribution of Eleven-lncRNA signature score (Figure 5B), survival status, and survival time (Figure $5 \mathrm{C}$ ) in the validation group were presented in Figure 5. The C-index of Eleven-lncRNA signature in validation group was 0.740 (95\% CI: $0.696-0.784)$ for overall survival.

\section{Clinical utility of Eleven-IncRNA signature in validation group}

In the validation group (Table $\mathrm{S} 2$ ), the $\mathrm{C}$-indexes of Eleven-lncRNA signature were $0.748,0.794$, and 0.798 for 1-year overall survival, 3-year overall survival, and 5 -year overall survival respectively (Figure 6A). A good agreement between predictive mortality probability and actual mortality percentage was found through calibration curves of 1-year overall survival (Figure 6B), 3-year overall survival (Figure 6C), and 5-year overall survival (Figure 6D).

Independence assessment of Eleven-IncRNA signature The independence of Eleven-lncRNA signature for overall survival was further explored by multivariate Cox regression analyses. In the present study, the pathological assessment was performed according to the criteria suggested by the American Joint Committee on Cancer (AJCC). As shown in Table 3, multivariate Cox regression analyses demonstrated that the prognostic significance of Eleven-lncRNA signature was independent to gender, age, AJCC PT, AJCC PN, AJCC PM, and AJCC stage.

\section{Subgroup analysis}

To explore the clinical application of Eleven-lncRNA signature, we further performed subgroup analysis in different AJCC stages (Figure 7). In GC patients with AJCC stage $1-2$, the overall survival rate (Figure 7A) in the high-risk group was significantly poorer than that in the low-risk 

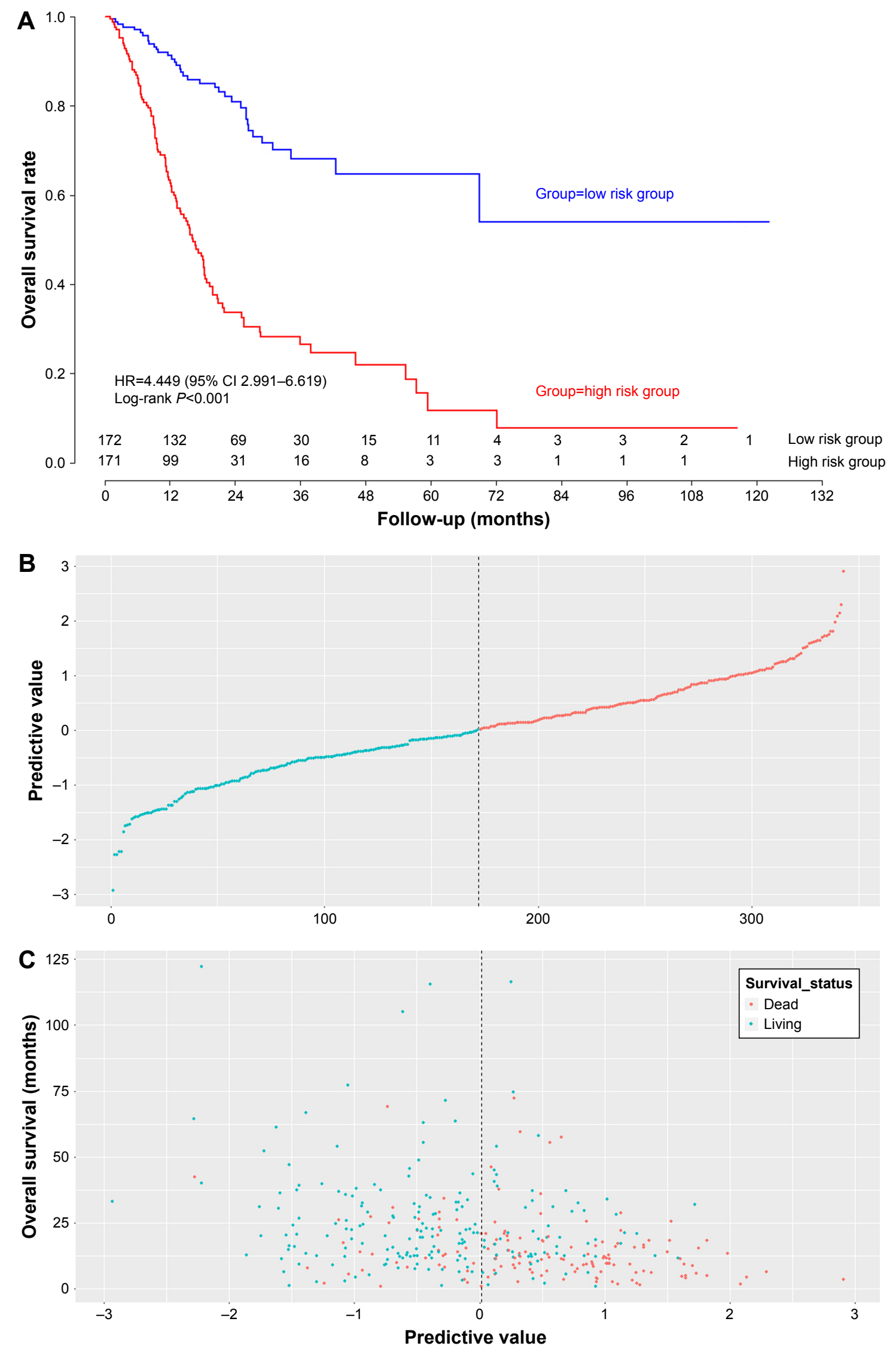

Figure 3 The survival curves of gastric cancer patients in model group.

Note: The survival curves in high risk group and low risk group (A), the distribution of Eleven-IncRNA signature scores (B), survival status and survival time (C) in the model group. Abbreviation: IncRNA, long non-coding RNA. 
A

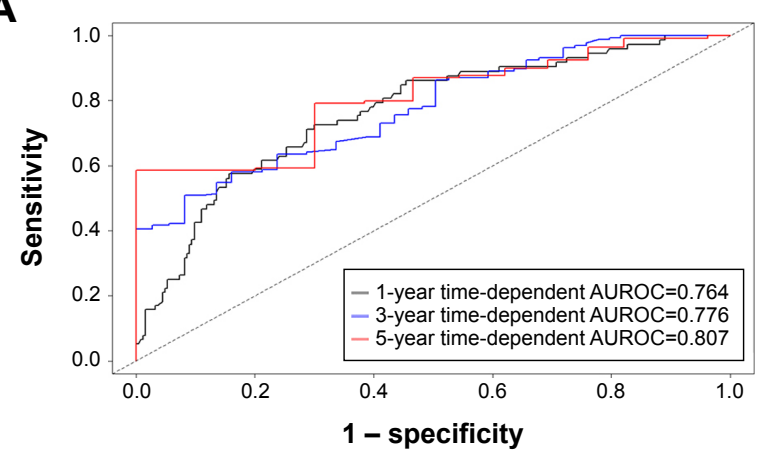

C

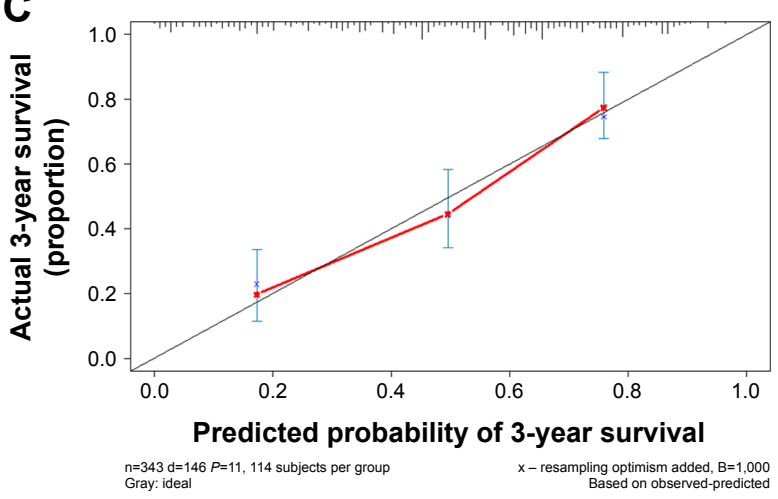

B

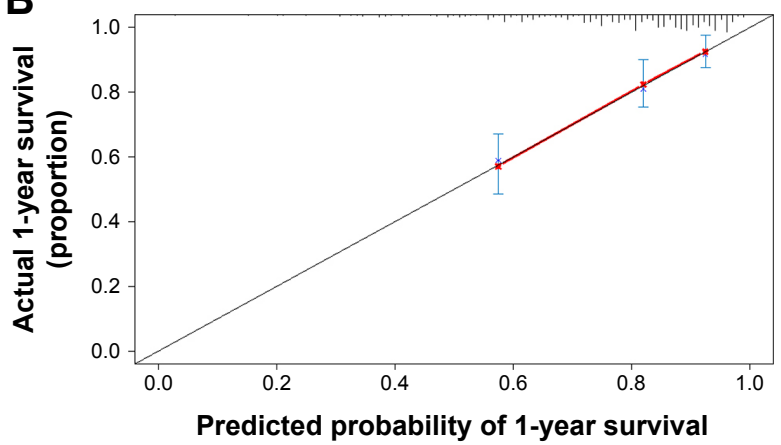

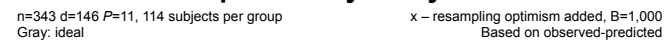

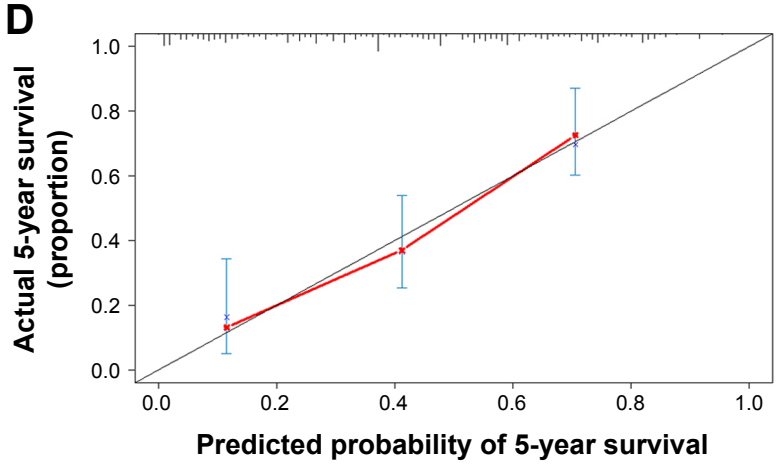
$\mathrm{n}=343 \mathrm{~d}=146 \mathrm{P}=11,114$ subjects per group
Gray:ideal

$\mathrm{x}$ - resampling optimism added, $\mathrm{B}=1,000$
Based on observed-predicted

Figure 4 Performance of Eleven-IncRNA signature in the model group.

Note: Time-dependent receiver operating characteristic curves (A); calibration curve for I-year overall survival (B); calibration curve for 3-year overall survival (C); calibration curve for 5-year overall survival (D).

Abbreviations: AUROC, area under the receiver operating characteristic curve; IncRNA, long non-coding RNA.

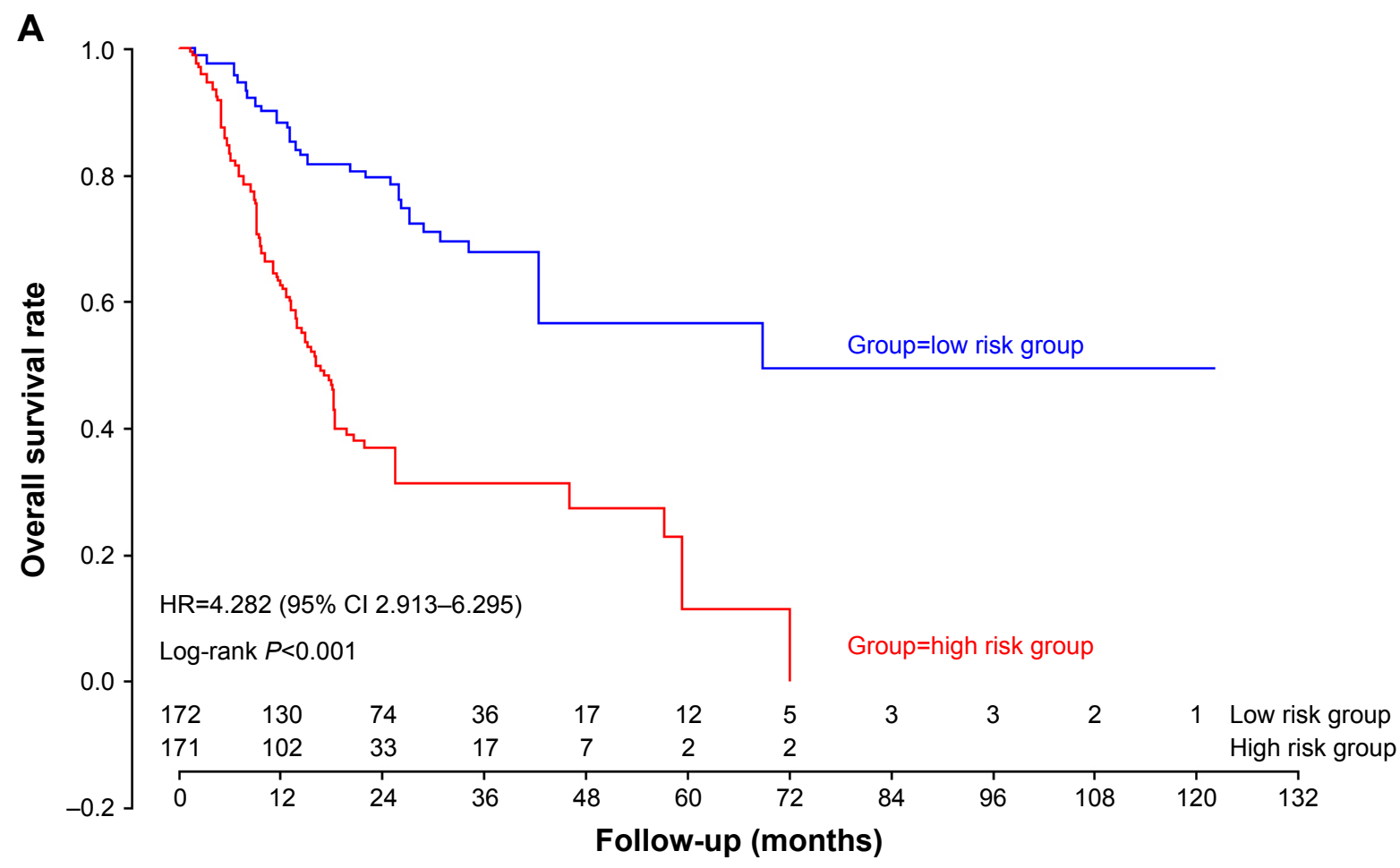

Figure 5 (Continued) 

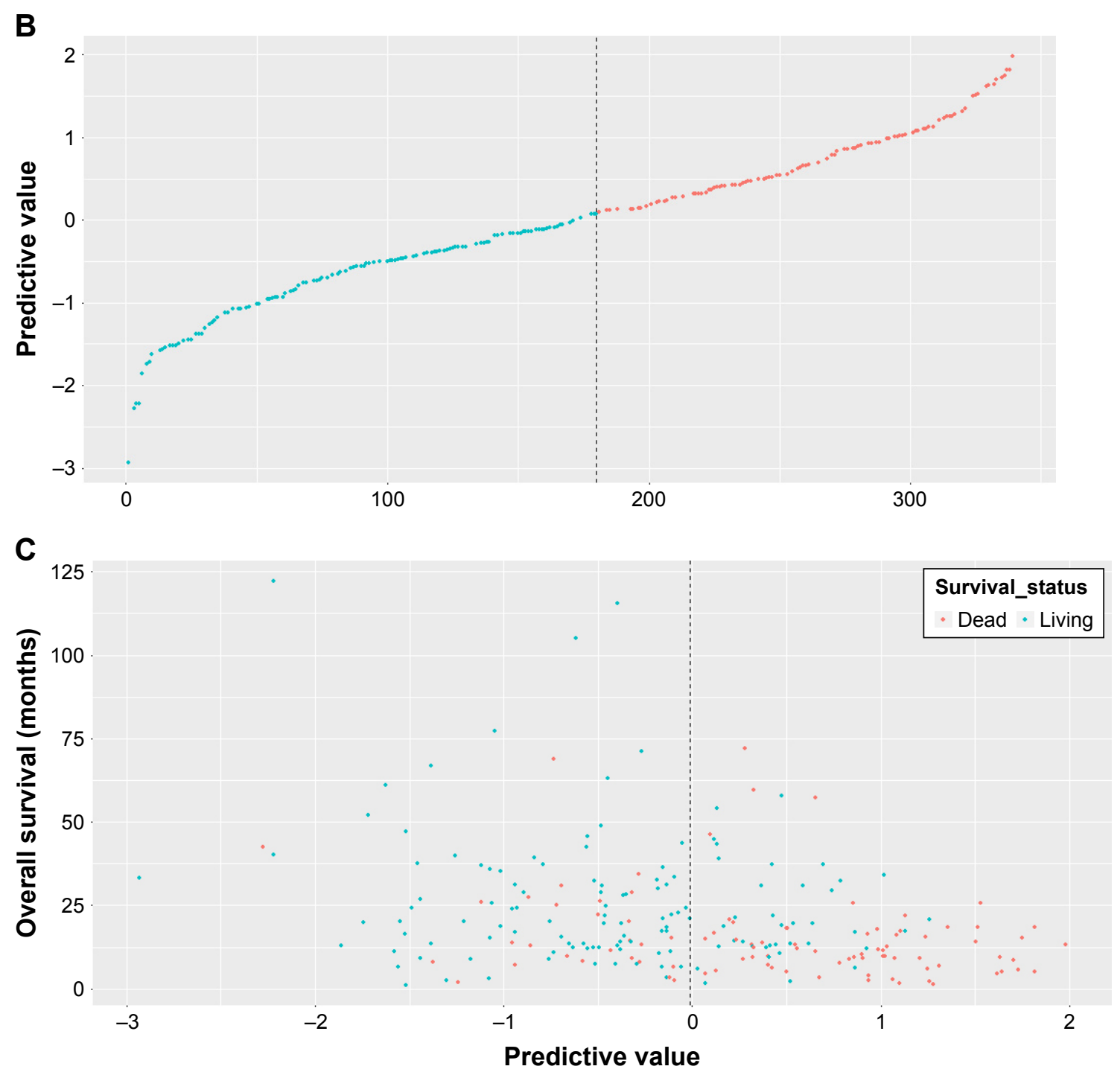

Figure 5 The survival curves of gastric cancer patients in validation group.

Note: The survival curves in high risk group and low risk group (A), the distribution of Eleven-IncRNA signature scores (B), survival status and survival time (C) in validation group. Abbreviation: IncRNA, long non-coding RNA.

group $(P<0.001)$. In GC patients with AJCC stage $1-2$, the C-indexes of Eleven-lncRNA signature were 0.766, 0.801, and 0.851 for 1 -year overall survival, 3-year overall survival, and 5-year overall survival respectively (Figure 7B).

In GC patients with AJCC stage 3-4, the overall survival rate (Figure $7 \mathrm{C}$ ) in the high-risk group was significantly poorer than that in the low-risk group $(P<0.001)$. In $\mathrm{GC}$ patients with AJCC stage 3-4, the C-indexes of ElevenlncRNA signature were $0.743,0.784$, and 0.884 for 1 -year overall survival, 3-year overall survival, and 5-year overall survival respectively (Figure 7D).

\section{Clinical association between IncRNA predictors and tumors}

Accumulated evidence has demonstrated that lncRNAs are involved in the progression of a wide variety of diseases.
To explore the clinical association between lncRNA predictors and tumors, we searched the potential associations between IncRNA predictors and tumors through MNDR version 2.0 database (http://www.rna-society.org/mndr/ index.html). According to the search results, three predictors (HOXA11-AS, ERICH3-AS1, and ZFPM2-AS1) out of eleven lncRNA predictors were associated with stomach cancer, hepatocellular carcinoma, and colorectal cancer with a confidence score of 0.1097 .

\section{Discussion}

We developed and validated a prognostic signature named Eleven-lncRNA signature, which might provide an effective individual mortality risk prediction and risk stratification in GC patients. The Eleven-lncRNA signature could help GC 

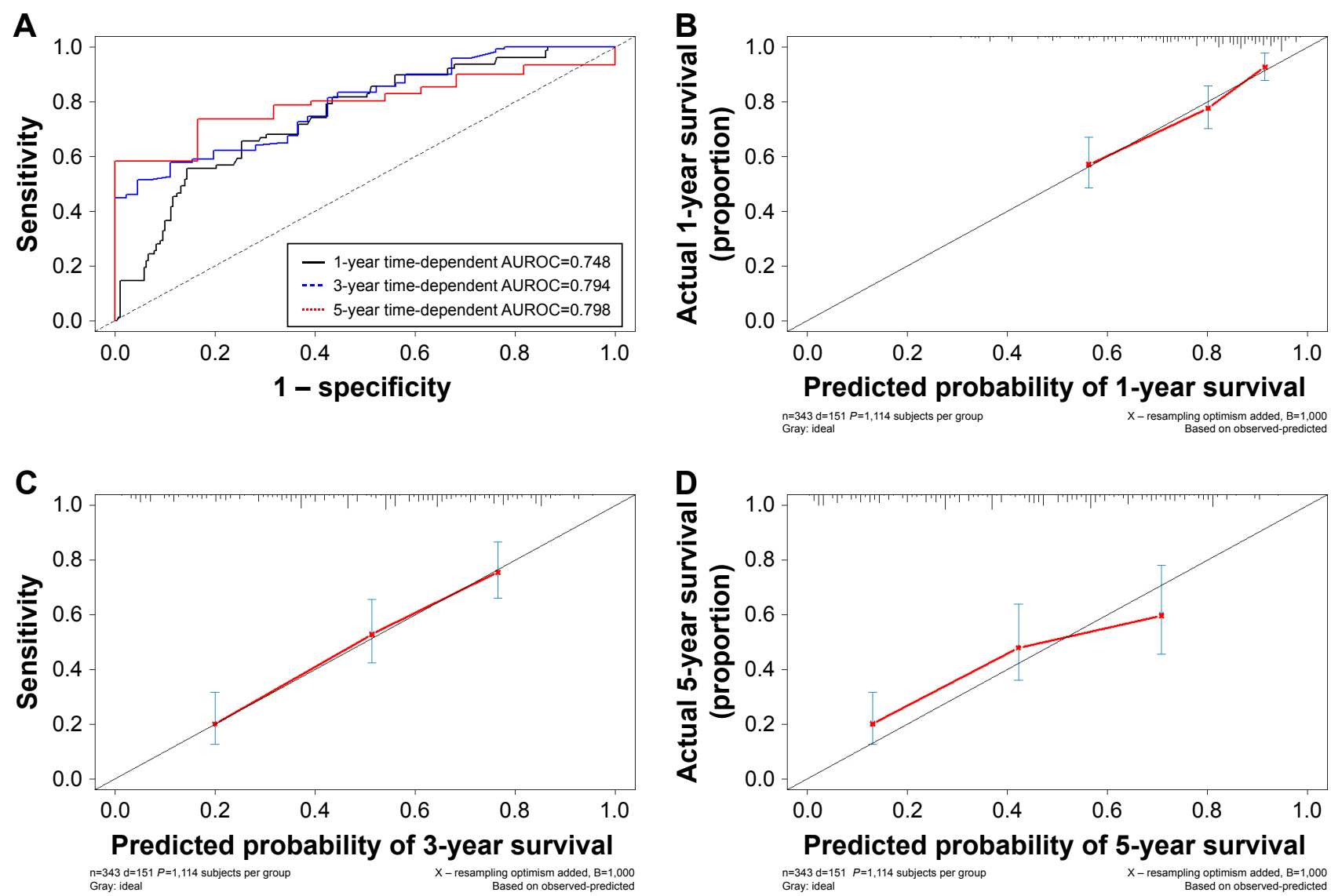

Figure 6 Performance of Eleven-IncRNA signature in validation group.

Note: Time-dependent receiver operating characteristic curves (A); calibration curve for I-year overall survival (B); calibration curve for 3-year overall survival (C); calibration curve for 5 -year overall survival (D).

Abbreviations: AUROC, area under the receiver operating characteristic; IncRNA, long non-coding RNA.

Table 3 Univariate and multivariable Cox regression analyses for independence assessment of Eleven-IncRNA signature

\begin{tabular}{|c|c|c|c|c|c|c|c|c|}
\hline & \multirow[t]{2}{*}{$\mathbf{N}$} & \multicolumn{3}{|c|}{ Univariate analysis } & \multicolumn{4}{|c|}{ Multivariate analysis } \\
\hline & & HR & $95 \% \mathrm{Cl}$ & $P$-value & Coefficient & HR & $95 \% \mathrm{Cl}$ & $P$-value \\
\hline \multicolumn{9}{|l|}{ Model group $(n=343)$} \\
\hline Age (high/low) & 343 & 1.504 & $1.085-2.086$ & 0.014 & 0.616 & 1.851 & $1.310-2.616$ & $<0.001$ \\
\hline Gender (male/female) & 343 & 1.370 & $0.960-1.957$ & 0.083 & 0.116 & 1.123 & $0.775-1.628$ & 0.540 \\
\hline AJCC PT (T4, T3/T2, TI) & 343 & 1.709 & I.133-2.577 & 0.011 & 0.316 & 1.372 & $0.823-2.286$ & 0.225 \\
\hline AJCC PN (N2, NI/N0) & 341 & 1.820 & $1.212-2.734$ & 0.004 & $0.44 I$ & 1.555 & $0.893-2.707$ & 0.119 \\
\hline AJCC PM (MX, MI/M0) & 343 & 1.895 & $1.181-3.039$ & 0.008 & 0.268 & 1.308 & $0.786-2.177$ & 0.302 \\
\hline AJCC stage (IV, III/II, I) & 328 & 1.794 & $1.260-2.555$ & $<0.001$ & 0.151 & 1.164 & $0.683-1.982$ & 0.577 \\
\hline $\begin{array}{l}\text { Eleven-IncRNA signature } \\
\text { score (high/low) }\end{array}$ & 343 & 4.253 & $2.922-6.191$ & $<0.00$ I & 1.493 & 4.449 & $2.991-6.619$ & $<0.001$ \\
\hline \multicolumn{9}{|l|}{ Validation group $(n=343)$} \\
\hline Age (years) & 343 & 1.340 & $0.972-1.848$ & 0.074 & 0.475 & 1.608 & I. $|44-2.26|$ & 0.006 \\
\hline Gender (male/female) & 343 & 1.620 & $1.129-2.325$ & 0.009 & 0.188 & 1.207 & $0.823-|.77|$ & 0.335 \\
\hline AJCC PT $(T 4, T 3 / T 2, T I)$ & 340 & 1.768 & $1.195-2.618$ & 0.004 & 0.337 & 1.400 & $0.869-2.255$ & 0.166 \\
\hline AJCC PN (N2, NI/N0) & 337 & 2.094 & $1.396-3.140$ & $<0.00$ I & 0.454 & 1.574 & $0.906-2.736$ & 0.107 \\
\hline AJCC PM (MX, MI/M0) & 340 & 1.631 & $0.955-2.786$ & 0.073 & 0.080 & 1.084 & $0.595-1.974$ & 0.793 \\
\hline AJCC stage (IV, III/II, I) & 321 & 1.756 & $1.24 \mathrm{I}-2.485$ & 0.001 & 0.196 & 1.216 & $0.737-2.007$ & 0.444 \\
\hline $\begin{array}{l}\text { Eleven-IncRNA signature } \\
\text { score (high/low) }\end{array}$ & 343 & 3.787 & $2.663-5.384$ & $<0.001$ & 1.455 & 4.282 & $2.913-6.295$ & $<0.001$ \\
\hline
\end{tabular}

Note: The median of Eleven-IncRNA signature scores was used as the cutoff value to stratify gastric cancer patients into high-risk group and low-risk group. Abbreviations: AJCC, American Joint Committee on Cancer; IncRNA, long non-coding RNA. 
A
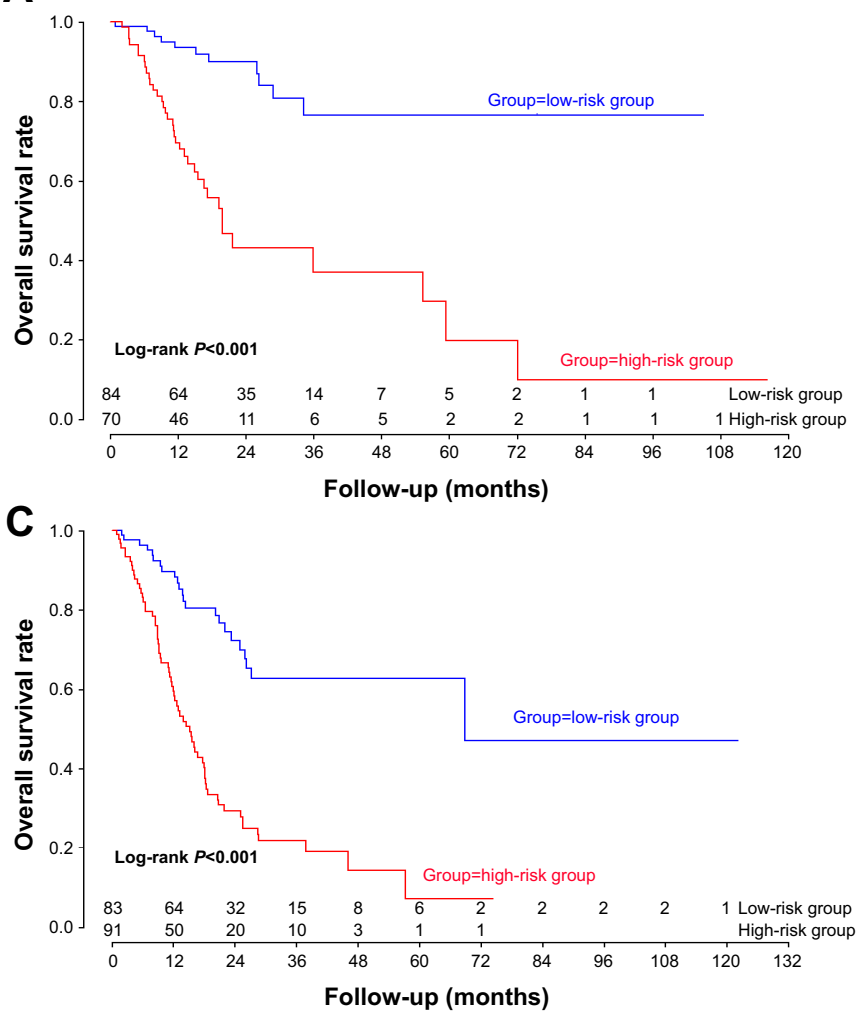

B

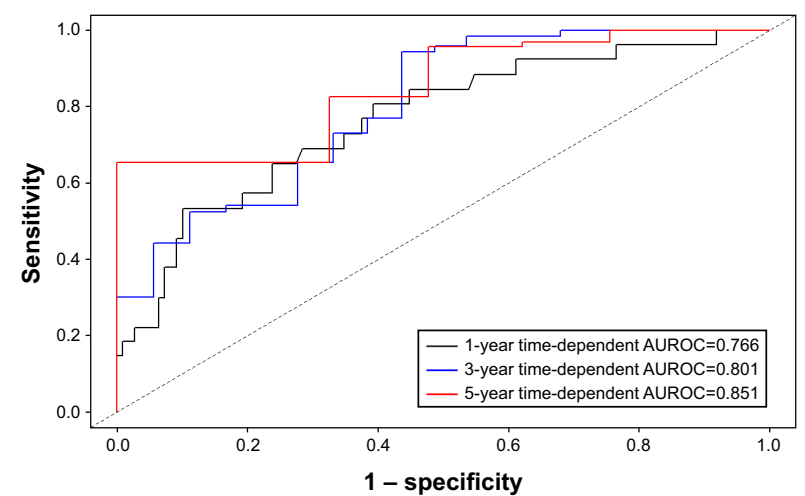

D

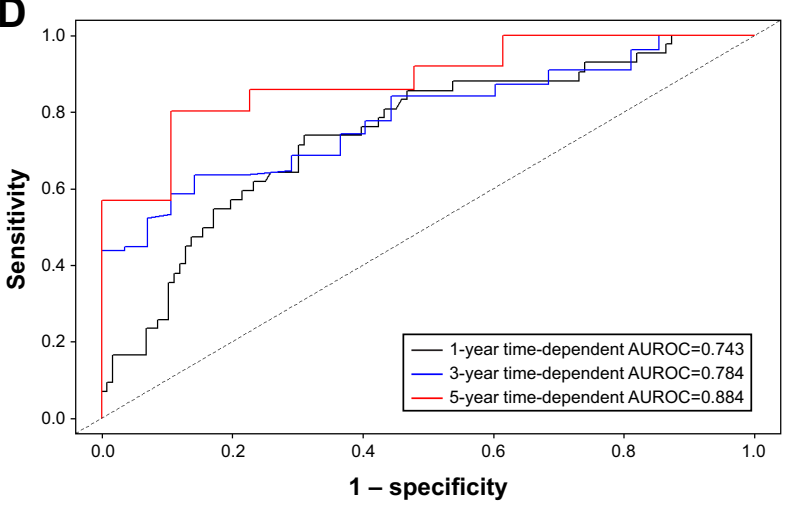

Figure 7 Subgroup analysis of the clinical application of Eleven-IncRNA signature in different AJCC stages.

Note: (A) The survival curves of gastric cancer patients with AJCC stage I-2; (B) time-dependent receiver operating characteristic curves of gastric cancer patients with AJCC stage I-2; (C) the survival curves of gastric cancer patients with AJCC stage 3-4; (D) time-dependent receiver operating characteristic curves of gastric cancer patients with AJCC stage 3-4.

Abbreviations: AJCC, American Joint Committee on Cancer; AUROC, area under the receiver operating characteristic; IncRNA, long non-coding RNA.

patients to predict their individual mortality risk and subsequently optimize their individual therapy decisions.

The Eleven-lncRNA signature was suitable for preoperative prediction of overall survival in GC patients for the following reasons: first, the Eleven-lncRNA signature was built based on IncRNA expression values without pathological parameters. Therefore the Eleven-lncRNA signature is a noninvasive preoperative predictive method. Eleven-lncRNA signature could serve as a convenient noninvasive preoperative screening method for GC patients, especially for those patients who could not tolerate surgery. Second, the original LncRNA expression values were converted to 1 for high expression and 0 for low expression according to the medians of original lncRNA expression values. The Eleven-lncRNA signature was calculated based on the converted dichotomous values but not the original lncRNA expression values. This conversion of original lncRNA expression values was helpful to improve the clinical applicability of Eleven-lncRNA signature on other platforms. Third, GC patients were stratified into high-risk group and low-risk group according to the median value of Eleven-lncRNA signature scores. The survival curves showed that the mortality rate of high-risk patients was significantly poorer than that of low-risk patients. The risk stratification method was helpful to monitor the patients with high mortality risk and select GC cancer patients who should receive active surgical treatment.

Due to the following reasons, the current study did not calculate previous prognostic signatures. ${ }^{11-14}$ First, these previous prognostic signatures were calculated based on the original gene expression values, which were generated on different gene detection platforms. The same sample would generate different expression values on different platforms. Therefore, the influence of different gene detection platforms should be taken into account during the development of prognostic signatures. Second, the lncRNA expression values were further standardized by different standardization methods, which might be not available for clinicians and patients. In order to overcome these two obstacles, we developed Eleven-lncRNA signature by using the converted dichotomous values but not the original lncRNA expression values. This conversion method of original lncRNA expression values was helpful to eliminate the effects of different 
detection platforms and standardization methods. Therefore, the Eleven-lncRNA signature was more suitable for clinical practice than previous prognostic signatures.

\section{Strengths and limitations}

There were several advantages in the present study: first, the Eleven-lncRNA signature can predict individual mortality probability, which is important in persuading GC patients to receive differentiated intensive treatment in time. Second, this Eleven-lncRNA signature can be calculated by patients without a special calculation tool. Third, the Eleven-lncRNA signature provides an alternative non-invasive preoperative prediction method for GC patients with an intolerance of surgical treatment. Therefore, as a non-invasive, simple, and comprehensible scoring model, the Eleven-lncRNA signature is suitable for preoperative prediction of overall survival of GC patients.

There were two disadvantages which must be taken into account in the present study. First, though an internal validation was carried out by using the bootstrap resampling method, external validation studies are needed to further validate the performance of Eleven-lncRNA signature in different populations. Second, as a retrospective study, the original clinical dataset did not provide additional information regarding medication status, postoperative treatment, and disease-specific survival. Thus, we could not explore the influence of medication status and postoperative treatment on overall survival. Meanwhile, the clinical application of Eleven-lncRNA signature for disease-specific survival was uncertain. Therefore, large prospective studies are needed to further elucidate the association between these prognostic IncRNA predictors and prognosis of GC patients.

\section{Conclusion}

The present study developed and validated a prognostic model named Eleven-lncRNA signature for preoperative, individual mortality risk prediction in GC patients. This Eleven-lncRNA signature can predict individual mortality risk of GC patients and is helpful in improving clinical decision making regarding individualized treatment.

\section{Acknowledgments}

We appreciate TCGA database for providing the original study data. This study was supported by Guangdong Provincial Health Department and Guangdong Provincial Financial Department. This study was supported by Guangdong Provincial Health Department and Guangdong Provincial Financial Department. The grant numbers were:
B2018237 (grant recipient: Zhiqiao Zhang) and A2016450 (grant recipient: Zhiqiao Zhang). The total capital was RMB 15,000 . The funders had no role in study design, data collection, analysis, decision to publish, or preparation of the manuscript.

\section{Author contributions}

Conceptualization: ZZ, ZL, KL, and MZ; data curation: ZZ and $\mathrm{QW}$; formal analysis: ZZ, MZ, and QW; funding acquisition: ZZ; investigation: ZZ, KL, and QW; methodology: ZZ and ZL; project administration: $Z Z, Z L$, and ZM; resources: ZZ and QW; software: ZZ and ZL; supervision: ZZ and MZ; validation: ZZ and QW; visualization: ZZ, QW, and ZL; writing original draft: ZZ, QW, and ZL; revising and editing: ZZ and QW. All authors contributed toward data analysis, drafting and critically revising the paper, gave final approval of the version to be published, and agree to be accountable for all aspects of the work

\section{Disclosure}

The authors report no conflicts of interest in this work.

\section{References}

1. van Cutsem E, Sagaert X, Topal B, Haustermans K, Prenen H. Gastric cancer. Lancet. 2016;388(10060):2654-2664.

2. Shah MA, Kelsen DP. Gastric cancer: a primer on the epidemiology and biology of the disease and an overview of the medical management of advanced disease. J Natl Compr Canc Netw. 2010;8(4):437-447.

3. Jung KW, Won YJ, Kong HJ, Oh CM, Shin A, Lee JS. Survival of korean adult cancer patients by stage at diagnosis, 2006-2010: national cancer registry study. Cancer Res Treat. 2013;45(3):162-171.

4. Cervantes A, Roda D, Tarazona N, Roselló S, Pérez-Fidalgo JA. Current questions for the treatment of advanced gastric cancer. Cancer Treat Rev. 2013;39(1):60-67.

5. Siegel R, Ma J, Zou Z, Jemal A. Cancer statistics, 2014. CA Cancer J Clin. 2014;64(1):9-29.

6. Marano L, Boccardi V, Braccio B, et al. Comparison of the 6th and 7th editions of the AJCC/UICC TNM staging system for gastric cancer focusing on the "N" parameter-related survival: the monoinstitutional NodUs Italian study. World J Surg Oncol. 2015;13:215.

7. Kim BS, Park YS, Yook JH, Kim BS. Comparison of the prognostic values of the 2010 WHO classification. AJCC 7th edition, and ENETS classification of gastric neuroendocrine tumors. Medicine. 2016; 95(30):e3977.

8. Mcfadden EJ, Hargrove AE. Biochemical methods to investigate IncRNA and the influence of lncRNA:Protein complexes on chromatin. Biochemistry. 2016;55(11):1615-1630.

9. Zhu X, Tian X, Yu C, et al. A long non-coding RNA signature to improve prognosis prediction of gastric cancer. Mol Cancer. 2016;15(1):60.

10. Tian X, Zhu X, Yan T, et al. Differentially expressed lncRNAs in gastric cancer patients: a potential biomarker for gastric cancer prognosis. J Cancer. 2017;8(13):2575-2586.

11. Ren W, Zhang J, Li W, et al. A tumor-specific prognostic long non-coding RNA signature in gastric cancer. Med Sci Monit. 2016;22:3647-3657.

12. Song P, Jiang B, Liu Z, Ding J, Liu S, Guan W. A three-lncRNA expression signature associated with the prognosis of gastric cancer patients. Cancer Med. 2017;6(6):1154-1164. 
13. Fan ZY, Liu W, Yan C, et al. Identification of a five-lncRNA signature for the diagnosis and prognosis of gastric cancer. Tumour Biol. 2016; 37(10):13265-13277.

14. Miao Y, Sui J, Xu SY, Liang GY, Pu YP, Yin LH. Comprehensive analysis of a novel four-lncRNA signature as a prognostic biomarker for human gastric cancer. Oncotarget. 2017;8(43):75007-75024.

15. Li Y, Xia Y, Li J, Wu D, Wan X, Wang K, Wu M. Prognostic nomograms for pre- and postoperative predictions of long-term survival for patients who underwent liver resection for huge hepatocellular carcinoma. J Am Coll Surg. 2015;221(5):962.e4-974.e4.

16. Collins GS, Reitsma JB, Altman DG, Moons KG. Transparent Reporting of a multivariable prediction model for Individual Prognosis or Diagnosis (TRIPOD): the TRIPOD Statement. Br J Surg. 2015;102(3): $148-158$.
17. Robinson MD, Oshlack A. A scaling normalization method for differential expression analysis of RNA-seq data. Genome Biol. 2010; 11(3):R25.

18. Blackstone EH. Breaking down barriers: helpful breakthrough statistical methods you need to understand better. J Thorac Cardiovasc Surg. 2001; 122(3):430-439.

19. Grunkemeier GL, Wu Y. Bootstrap resampling methods: something for nothing? Ann Thorac Surg. 2004;77(4):1142-1144.

\section{Publish your work in this journal}

OncoTargets and Therapy is an international, peer-reviewed, open access journal focusing on the pathological basis of all cancers, potential targets for therapy and treatment protocols employed to improve the management of cancer patients. The journal also focuses on the impact of management programs and new therapeutic agents and protocols on

\section{Dovepress}

patient perspectives such as quality of life, adherence and satisfaction. The manuscript management system is completely online and includes a very quick and fair peer-review system, which is all easy to use. Visit http://www.dovepress.com/testimonials.php to read real quotes from published authors. 